\title{
La réhabilitation idéologique post-stalinienne de l'architecture vernaculaire
}

Post-Stalin Ideological Rehabilitation of Vernacular Architecture

\section{Augustin loan}

\section{CpenEdition}

Journals

Édition électronique

URL : http://journals.openedition.org/etudesbalkaniques/116

ISSN : 2102-5525

\section{Éditeur}

Association Pierre Belon

\section{Édition imprimée}

Date de publication : 1 janvier 2005

Pagination : 187-189

ISBN : 2-910860-05-1

ISSN : $1260-2116$

\section{Référence électronique}

Augustin loan, « La réhabilitation idéologique post-stalinienne de l'architecture vernaculaire », Études balkaniques [En ligne], 12 | 2005, mis en ligne le 07 avril 2009, consulté le 08 mai 2019. URL : http:// journals.openedition.org/etudesbalkaniques/116

Ce document a été généré automatiquement le 8 mai 2019.

Tous droits réservés 


\title{
La réhabilitation idéologique post- stalinienne de l'architecture vernaculaire
}

\author{
Post-Stalin Ideological Rehabilitation of Vernacular Architecture
}

\section{Augustin loan}

1 Au lendemain de l'épisode stalinien roumain, dans le contexte politique d'une volonté d'éloignement de Moscou des communistes roumains, l'architecture vernaculaire est de nouveau courtisée par les architectes. Ils la considèrent comme une possible source d'inspiration « oubliée » ou, plus précisément, idéologisée à l'excès. Les informations que ces architectes m'ont fournies au cours des interviews qui ont soutenu ma recherche, comme les textes publiés pendant cette période montrent que l'architecture "populaire », vernaculaire, était considérée comme « de gauche ». Dans le débat sur la manière dont on allait édifier après le stalinisme des édifices représentatifs, un choix était proposé entre l'architecture byzantine -comme celle promue par l'architecte Simotta au Palais métropolitain, ou celle, revisitée dans un esprit moderne, par l'équipe qui a réalisé l'Ecole Polytechnique à Bucarest- et l'architecture vernaculaire ou paysanne. La seconde a gagné, plutôt pour des raisons idéologiques : le vernaculaire devient une possible source de "rationalité », voire de modernité dans la perspective du discours khrouchtchevien : efficacité dans l'utilisation des matériaux, sobriété dans la décoration plaident en sa faveur. Par conséquent, il peut irriguer une fois de plus le discours architectural « urbain » qui avait momentanément oublié ses racines naturelles. ${ }^{1}$

C'est dans un esprit similaire conjuguant modernité et archaïsme, puisque l'archaïque est redécouvert comme une possible source de modernité, qu'a été créé, entre 1955 et 1957, le petit lotissement d'habitations sociales du quartier Catelu adjacent à l'avenue Mihai Bravu de Bucarest. Tiberiu Niga, un architecte distingué de l'entre-deux-guerres, fit bénéficier le projet de Catelu de son intérêt jamais démenti pour l'architecture "populaire » roumaine, une architecture redevenue politiquement correcte à l'époque, grâce à la reconnaissance du caractère « de gauche » du vernaculaire, qui le faisait sortir, 
au moins pour une courte période, d'un contexte d'orientations nationalistes, toujours considérées "de droite", sinon purement "fascistes ». La justification du lotissement d'habitations « d'urgence » Catelu/Mihai Bravu est double : d'une part, les appartements d'une seule ou de deux pièces tireraient leur origine de « notre demeure traditionnelle $»^{2}$, d'autre part le lotissement reprendrait les thèmes communs aux architectures expérimentées dans le domaine - on ne précise pas lesquelles, mais le contexte indique qu'il ne s'agit pas de celles du camp socialiste. Le traitement plastique vise à l'obtention d'une "architecture humaine " ${ }^{3}$, avec tous les traits que nous reconnaissons au canon moderne : "la simplicité des formes, l'expression sincère des fonctions", des traits qui sont aussi à identifier comme étant propres à «notre architecture traditionnelle $»^{4}$. La conclusion, vers laquelle paraissent converger tous les arguments du chef de projet, l'auteur de l'ensemble Catelu, est que la modernité est consubstantielle au "caractère propre de notre architecture $»^{5}$. C'est une idée qui, reprise et gonflée démesurément par le discours du politique, sera, théorisée deux décennies plus tard par Constantin Joja comme attribut de la spécificité nationale roumaine : l'invention de l'architecture moderne.

3 Mais pour l'heure on n'en est pas là. Pour comprendre le climat il suffit de noter le commentaire de Grigore Ionescu sur l'ensemble qu'il estime avoir un «caractère autochtone accentué $»^{6}$, bien qu'on puisse observer que dans l'architecture locale vernaculaire on trouve peu « de portiques, de loggias, de galeries et d'escaliers extérieurs recouverts $»^{7}$ qu'on pourrait invoquer comme précédents. Il est vrai que des proportions analogues à celles de l'» ancienne architecture roumaine $»^{8}$ peuvent être relevées, surtout si l'on compare ce petit quartier aux ensembles qu'on construisait déjà au centre-ville, ou, sur le Boulevard Magheru: les immeubles post-staliniens s'élevaient aux proportions d'avant-guerre, avec huit et dix étages. Le quartier de Catelu est pittoresque, il est ingénieusement articulé avec des espaces semi-publics, des rues et des cours intérieures où les enfants peuvent jouer en toute sécurité sous le regard attentif, on dirait aujourd'hui panoptique, des mères de la communauté, et des espaces semi-privés, des terrasses qui servent autant pour l'accès à la maison que pour une socialisation de proximité. Son architecture domestique, calme, paraît très éloignée du tumulte idéologique et esthétique de l'époque qui l'a rendue possible. Par la suite, la simplicité de l'exécution l'a recommandée comme un prototype plausible pour les habitations collectives d'urgence, encore nécessaires en Roumanie.

4 Malheureusement, l'état actuel du quartier n'est plus celui de ses origines. La propriété privée s'est emparée de portions massives de l'espace communautaire, elle a fermé les tracés d'ensemble, parcourus furtivement par les bandes d'enfants des premiers habitants: les émigrants bessarabiens. Surtout, l'évolution a altéré l'architecture originale. Les mémoires ${ }^{9}$ des architectes distingués qui ont grandi dans ces quartiers marginaux, au propre (par rapport géographique au centre-ville), comme au figuré (par rapport au courant idéologique dominant de l'époque), de même que la redécouverte de la pluralité des modernismes nous incitent à remettre en discussion la valeur aujourd'hui incontestable- de ces ébauches d'architecture de haute qualité. Insolites pour leur époque, elles prouvent aujourd'hui, au contraire, leur «normalité ». De nos jours, la conservation, du corpus d'architecture stalinienne doit avoir comme corollaire la préservation, des « écarts ", si modestes et relatifs soient-ils, au dogme. Le classement du quartier Catelu dans le patrimoine architectural de Bucarest et, par suite, sa préservation, 
serait non seulement un geste noble pour son indiscutable qualité urbaine et architecturale, mais aussi une occasion d'en arrêter la dégradation continue.

\section{NOTES}

1. «L'architecture populaire, étant l'architecture des villages, est un produit de la culture paysanne ; le paysan a une mentalité propre, qui se reflète dans toutes ses réalisations et qui se caractérise par un esprit pratique et économe. Ces traits le font aborder les problèmes de construction sans le romantisme de certains citadins, dont les aspirations en ce qui concerne leurs maisons se rapportent surtout à un faux décor stylistique », R. Crainiceanu, "Nouvelles maisons sur la Voie du Jiu », Architectura, n. 9 (1957).

2. T. Niga, «Un quartier d'habitations minimales dans Bucarest », Arhitectura RPR, n. 2 (1957), p.

3. La présentation de l'ensemble en détail est à trouver aux pages 3-11 dans la même revue.

3. Ibid., p. 5

4. Ibid.

5. Ibid.

6. G. Ionescu, L'Architecture sur le territoire de la Roumanie le long des siècles, Bucarest, 1980, p. 643.

7. Ibid.

8. Ibid.

9. F. Biciusca, L'Expérimentation Catelu: Bucuresti : Liternet/Paideia (e-book et livre imprimé), Collection « Poverism » sponsorisée par la Fondation Habitat et Art en Roumanie, 2005.

\section{RÉSUMÉS}

L'auteur se penche sur la "réhabilitation » du vernaculaire, en tant que source d'inspiration, après l'épisode stalinien qu'avait subi l'architecture roumaine. Il analyse les vertus qu'on lui attribuait à l'époque - architecture "de gauche ", mais aussi éloignée des excès idéologiques du réalisme socialiste - en prenant comme étude de cas un petit lotissement d'habitations sociales de Bucarest, construit entre 1955-1957.

After a Stalinist period, Rumanian architecture has been renewed by the "rehabilitation" of vernacular as a source of inspiration. The author analyzes the values which have been attributed at that time to a "left-wing" - but far from ideological excesses of socialist realism - architecture. By way of illustration he gives a case study on a little estate for social housing in Bucharest, built between 1955-1957. 
AUTEUR

AUGUSTIN IOAN

Université d'Architecture, Bucarest 